\title{
Big Bang Scenario and Nature of Dark Matter
}

\author{
I. Novikov ${ }^{1,2 * 3,4}$
}

1) Astro Space Center of P.N.Lebedev Physical Institute Profsoyuznaya 84/32, Moscow, 117810, Russia

2) University Observatory, Øster Voldgade 3, DK-1350 Copenhagen K, Denmark 3) NORDITA, Blegdamsvej 17, DK-2100 Copenhagen, $\varnothing$ Denmark

4) Theoretical Astrophysics Center, Blegdamsvej 17, DK-2100,

Copenhagen $\varnothing$, Denmark

*) Permanent address

\begin{abstract}
We give a brief review of a problem of dark matter in the Universe. The key questions regarding dark matter are discussed: what is the distribution of dark matter at different scales? What is the nature of dark matter? Is there baryonic dark matter? We discuss a few new techniques of the investigation of dark matter in the Universe. We discuss the peculiar periodic dependence of the initial spectrum of the baryonic matter distribution on wavelength at the moment of recombination (Sakharov oscillations). Sakharov oscillations should manifest themselves by the specific anomalies in the angular correlation function of the microwave background anisotropy on scales $10^{\prime}-2^{\circ}$, practically in any cosmological model. We discuss special methods for filtering of this effect in the observational data.
\end{abstract}

\section{Introduction}

First International Astronomical Union Symposium devoted to the problem of Dark Matter in the Universe was held in 1985, but the history of the problem began much earlier. F. Zwicky (1933) was the first to point out that the sum of masses of visible galaxies in Coma Clusters is essentially less than the total mass of this cluster producing the gravitational field, assuming that cluster is a gravitationally bound and well relaxed system, and the virial theory can be 
applied. He concluded that either the cluster is short lived or visible matter is not a good guide for the mass. Thus invisible mass must exist in this cluster.

Another evidence about dark matter came in the 1970's from observations of rotation curves (dependence of the rotational Kepler's velocity on radius $r$ ) of spirals.

Nowadays the problem of dark matter in the Universe is more than 60 years old and yet is at the heart of all astrophysics and physics. This is not a surprise. The mass of the dark matter dominates in the Universe, determines the dynamics of the Universe and the quervature of the 3-dimensional space. On the other hand the dark matter plays a crucial role in the process of growth of small fluctuations in the matter distribution (progenitors of galaxies), in the processes of the origin of the large scale structure in the Universe, in the process of galaxy formation and evolution. The last but not list, dark matter determines the physical contents of the Universe and carries the information about the physics of the Early Universe.

In the Preface of the Proceedings of the 117 IAU Symposium, J.Kormendy and G.R.Knapp wrote: "This is the first time that the International Astronomical Union has held a symposium on objects of totally unknown nature" (Kormendy and Knapp, 1987). Unfortunately, today we have to repeat that the nature of dark matter is still unknown.

\section{Dark matter on different scales}

What can one say about the amount of various types of matter in the Universe? Surveys of visible matter (visible parts of galaxies, hot gas etc.) get (in dimensionless units $\left.\Omega \equiv\langle\rho\rangle / \rho_{\text {crit }}\right)$

$$
\Omega_{v i s} \approx 0.003-0.006,
$$

see for example Persic and Salucci (1992), Steigman (1994). On larger and larger mass/length scales the value $\Omega$ increases. If one considers only the visible parts of galaxies then the value of $\Omega$ is given by (1). However, on the scale of 1-3 hundred $\mathrm{Kpc}$ (scales of spiral halos and small groups of galaxies), the value of $\Omega$ has increased and $\Omega_{\text {Halo }} \approx 0.01-0.09$ (Griest (1994), Steigman (1994)). This trend continues up to the scale of rich clusters and beyond. On the scales of clusters $\Omega_{c l} \approx 0.1-0.3$ (Steigman (1994), Bahcall (1994)). The velocity distribution of galaxies on very large scales (large scale flows) provide the probes of the largest scales. A. Dekel (1993), (1994) concludes that the velocity data indicates a high value for $\Omega_{L S F}$ near unity, with $\Omega_{L S F} \leq 0.3$ strongly ruled out.

It is worth noting the following three new techniques of the investigation of missing mass (dark matter) in the Universe.

First of them is the amplification of light from distant stars as they crossed very near to the line-of-sight due to the relativistic microlensing effect. This 
method was proposed by Paczynski (1986) to detect Massive Astrophysical Compact Halo Objects (MACHOs). A few events were detected (Alcook et al. (1993), Aubourg et al. (1993)) from photometric measurements of millions of stars in the Large Magellanic Cloud. Probably MACHOs have masses of about 0.1 solar masses. The nature of them is not known. It is still unclear if the total number of MACHOs is enough to be halo dark matter.

Second technique is mapping cosmic dark matter in clusters of galaxies via the gravitational lens technique (Tyson (1994)). Foreground mass concentrations in a cluster of galaxies can be detected and mapped directly by the way they distort images of background sources. One can use as sources QSOs or far galaxies. QSOs are not so good due to the relative scarcity of QSOs compared with galaxies at similar redshifts. Thus they can give a little information on the mass distribution in the lens. Tyson et al. (1990) and Tyson (1994) have pointed out that faint blue galaxies could be excellent background sources permitting the tomographic mapping of the projected dark matter distribution.

The third technique is so called POTENT reconstruction (see Dekel (1994)). The POTENT method recovers the smoothed dynamical fluctuation fields of potential velocity and mass density from observed radial peculiar velocities of galaxies under quasilinear gravitational instability.

\section{The nature of dark matter}

What can one say about nature of dark matter? There are strong restrictions on the possible average baryon density, $\Omega_{b}$, from the comparison of the predictions of the theory of Big Bang nucleosynthesis with the observational abundances of the light elements. Big Bang nucleosynthesis calculations predict the primordial abundances of the light nucleides. The predictions depend on the baryon abundance and, consistency with observation is found for

$$
0.010 \leq \Omega_{b} h^{2} \leq 0.016
$$

where $H_{0}=100 \mathrm{kms}^{-1} \mathrm{Mpc}^{-1}$, see Olive et al. (1990), Pagel (1990), see also Steigman and Tosi (1992), the analysis of new data see Pagel (1994 a,b,c) analysis of the new observation of D/H ratio by Songalia et al. (1994) and Carswell et al. (1994) see Pagel $(1994$, a). Thus we get

$$
\Omega_{b}>\Omega_{v i s}
$$

suggesting that perhaps most baryons in the universe are dark. Part of this dark baryons could be in the form of hot gas in the rich clusters of galaxies. This gas is optically dark but visible in x-rays. Another part may be hiding in the halos of galaxies. 
The data on clusters and large scale flows suggests $\Omega_{\text {Halo }}, \Omega_{L S F} \geq 0.1-0.3$. Thus we get

$$
\Omega_{\text {Halo }}, \Omega_{L S F}>\Omega_{b} \text {. }
$$

The conclusions that most of the mass in the Universe must be non-baryonic. What is it then?

The nature of the non-baryonic dark matter is not known. The formation of the large scale structure in the Universe depends on some general properties of dark matter candidates. The analysis of the large scale structure formation is the main probe for the investigation of the nature of the dark matter. According to the convenient classification the dark matter candidates are divided into two categories: "Hot Dark Matter" (HDM) and "Cold Dark Matter" (CDM). At present various types of the large scale formation models are discussed: HDM, CDM, "Mixed" (i.e. with the following content: baryons $\Omega_{b}=0.05$, HDM $\Omega_{H D M}=0.25$ and CDM $\left.\Omega_{C D M}=0.70\right)$. Other possibilities are to include in the consideration the cosmological constant $\Lambda$ or topological defects such as cosmic strings.

What could be the suspect weakly-interacting massive particles left over from earliest moments of the Universe and which now form the dark matter? Massive neutrinos are the favored HDM candidates, a favored CDM candidate is the axion. The modern physics provides many excellent other candidates (see Kolb and Turner (1990)), but I have to repeat that we know practically nothing about the physical nature of dark matter.

\section{Sakharov oscillations}

In the subsequent part of the paper we will discuss one more technique of the investigation of the nature of dark matter. This technique was discussed in our papers Naselsky and Novikov (1993a,b) and Jørgensen et al. (1994). I'll follow the last of these papers.

Let us consider the transitional phenomena which take place at the epoch of recombination of cosmic plasma in the expanding Universe. Sakharov (1965) has been the first to realize that the transitional phenomena in the growing perturbations lead to a peculiar periodic dependence of the perturbation amplitudes on wavelength. He considered the cold model of the Universe, though, qualitatively speaking, the same phenomena occur in the hot model as a result of recombination. These Sakharov oscillations have been discussed, for example, by Peebles and Yu (1970), Zeldovich and Novikov (1983) in the framework of the baryonic model with adiabatic initial perturbations.

It should be emphasized that in any cosmological model at the time of recombination $\left(z_{\text {rec }} \simeq 1100\right)$ there existed acoustic modes of perturbations in the cosmic plasma due to the interaction between the baryonic matter and radiation. The wavelengths of the modes correspond to the comoving linear scales 
between the Silk damping scale, $l_{s} \sim 10 h^{-1} \mathrm{Mpc}$, and the acoustic horizon $R=$ $r_{\text {rec }} \frac{v_{\text {acoust }}}{c} \approx 100-200 h^{-1} \mathrm{Mpc}, r_{\text {rec }}$ is the event horizon at $z_{\text {rec }} \simeq 1100$, all being scaled to the present epoch, $v_{\text {acoust }}=3^{-1 / 2} / \sqrt{1+3 \rho_{b} / 4 \rho_{r}}$ where $\rho_{b} / \rho_{r}$ is the ratio of baryon and radiation densities at $z_{\text {rec }}$. After recombination the motion of baryonic matter is independent of the background radiation.

In non-baryonic dark matter models (for example - CDM models) the baryonic density fluctuations follow that of the dark matter, due to the peculiar gravitational field of the dark matter, whereas the distribution of the microwave background photons gets frozen on the celestial sphere thus preserving the information about the inhomogeneities at the last scattering surface. Therefore, in the present spatial distribution of matter, which is determined mainly by that of the dark matter, acoustic modes are practically erased, while the angular distribution of CMB preserves the signature of the acoustic motions of matter.

Now many groups observe the anisotropy of CMB on the scales of the order $1^{\circ}$. Because the upper limit on the scale of acoustic perturbations, $R$, is determined only by $r_{r e c}$ and $\rho_{b} / \rho_{r}\left(z_{r e c}\right)$, it is clear that detecting the effects of these modes in $\Delta T / T(\theta)$ would give unique information on the amount of baryons in the Universe as well as the spectrum of density perturbations on scales around $r \simeq 100 h^{-1} \mathrm{Mpc}$. The analysis of the manifestation of the Sakharov's oscillations see, for example, in the papers Holtzman (1989), Muciaccia et al. (1993). Authors use another names for this effect: "Doppler peak" for example.

\section{Filtering of Sakharov effect in the anisotropy of CMB.}

In the papers by Naselsky and Novikov (1993a,b) and Jørgensen et al. (1994) we investigated the manifestation of Sakharov oscillations in the anisotropy of Cosmic Microwave Background (CMB). We called the corresponding peculiarities of the angular correlation function of $\Delta T / T$ Long Distance Correlations (LDC). The LDC are thus a specific signature of the acoustic modes.

In the paper Naselsky and Novikov $(1993 \mathrm{a}, \mathrm{b})$ it was demonstrated that at the angular scales $\theta \approx \theta_{R}$ the Sakharov oscillations lead to an appearance of a specific anomaly (a "resonance" type) which has a half-width of order $\theta_{c}$, where $\theta_{R}$ is equal to the ratio of the acoustic horizon $R$ at the epoch of recombination (scaled to the present epoch) to the present-day particle horizon $h$, and $\theta_{c}=l_{s} / h, l_{s}$ is the Silk damping scale.. An analogous feature occurs at $\theta \rightarrow 2 \theta_{R}$. The existence of the feature at $\theta \approx 2 \theta_{R}$ for the limit case $\Omega_{b} \rightarrow 0$ was mentioned in the paper of Starobinskii (1988).

We would like to emphasize that the anomalies at $\theta \approx \theta_{R}$ and $\theta \approx 2 \theta_{R}$ have similar nature. The reason for both of them is the existence of acoustic modes evolving in the cosmic plasma at the epoch of hydrogen recombination. 


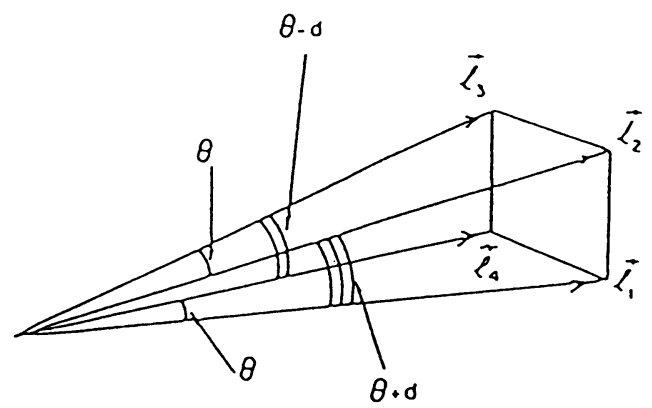

Figure 1: Space orientation of the unit vectors for the 4-beams experiment.

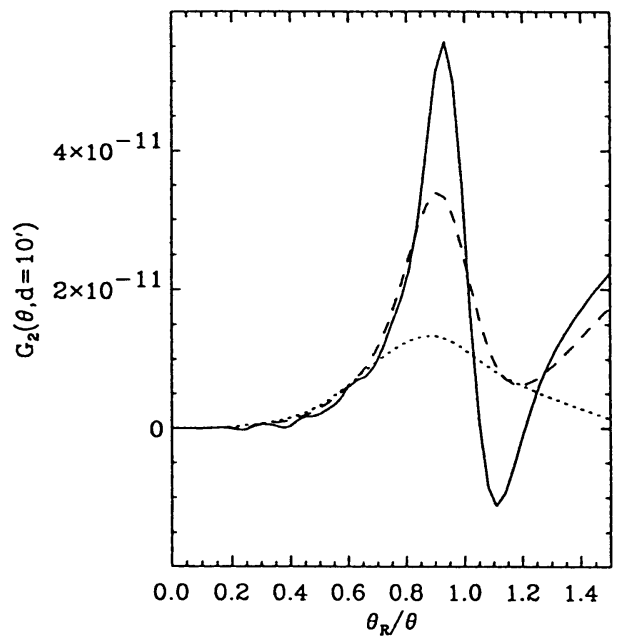

Figure 2: Function $G_{2}\left(\theta, d, \theta_{A}\right)$ for the model $\Omega=1 ; \Omega_{b}=0.03 ; h=0.5 ; d=10^{\prime}$ and the Harrison -Zel'dovich spectrum. Solid line for $\theta_{A}=0$, dashed line for $\theta_{A}=0.1 \theta_{R}$, dotted line for $\theta_{A}=0.3 \theta_{R}$. 
These acoustic modes left traces on the correlation function of the CMB angular fluctuations due to Silk (1968) and Doppler effects.

It is important to emphasize the following fact. In the "resonance" $\theta \sim \theta_{R}$ the Silk effect only gives the contribution, while in the "resonance" $\theta \approx 2 \theta_{R}$ both effects (Silk and Doppler) make their contributions. Thus we have an unique possibility of an experimental test of these effects, which are important both for the clarification of the character of the initial perturbations and for the investigation of the ionization history of the cosmic plasma.

What could be the method of filtering of the microwave background anisotropies which 1) cancels the regular component of the correlations and 2) emphasizes/preserves the effect of LDC? One method is to investigate the multipole moments representation of the angular correlation function

$$
C(\theta)=\langle T(\alpha) T(\alpha+\theta)\rangle_{\alpha}=\frac{1}{4 \pi} \sum_{l}(2 l+1) C_{l} P_{l}(\cos \theta),
$$

where $C_{l}$ are multipole moments, see, for example Smoot (1994).

We proposed another method (see Naselsky and Novikov (1993a,b)) which is an analogy of the method used by Watson and Gutierrez de la Cruz (1993): the observer should measure the difference of intensity of CMB in $\boldsymbol{l}_{1}$ and $\boldsymbol{l}_{3}$ directions, and in $\boldsymbol{l}_{2}$ and $\boldsymbol{l}_{4}$ as it is shown on Fig.4. We introduce an auxiliary function of two variables $\theta, d$ for given $\theta_{A}$ as:

$$
\begin{aligned}
& G_{2}\left(\theta, d, \theta_{A}\right)=\frac{\left\langle\left[T\left(l_{1}\right)-T\left(l_{3}\right)\right]\left[T\left(l_{2}\right)-T\left(l_{4}\right)\right]\right\rangle}{T^{2}}= \\
& =C\left(\theta+d, \theta_{A}\right)+C\left(\theta-d, \theta_{A}\right)-2 C\left(\theta, \theta_{A}\right),
\end{aligned}
$$

where $\boldsymbol{l}_{1} \cdot \boldsymbol{l}_{4}=\boldsymbol{l}_{2} \cdot \boldsymbol{l}_{3}=\cos \theta, \boldsymbol{l}_{1} \cdot \boldsymbol{l}_{2}=\cos (\theta+d), \boldsymbol{l}_{3} \cdot \boldsymbol{l}_{4}=\cos (\theta-d), T$ is the average temperature, $\theta_{A}$ is the half-width of the antenna-beam. The value of $\theta$ should vary somewhat more than the range $\theta_{R} \leq \theta \leq 2 \theta_{R}$, and $d \leq \theta_{c}$. The method can be used both for the direct measurement with the 4-beam experiment as well as the specific data-reduction technique.

One can see from (6) that for $d \ll \theta$ the level of anisotropy is related to the second derivative of the correlation function $C^{\prime \prime}\left(\theta, \theta_{A}\right)$ via:

$$
G_{2}\left(\theta, d, \theta_{A}\right) \simeq C^{\prime \prime}\left(\theta, \theta_{A}\right) d^{2} .
$$

We show that the quantity $G_{2}\left(\theta, d, \theta_{A}\right)$ introduced above allows one to measure in an optimal way the contribution of acoustic modes to the anisotropy of CMB.

On Fig. 2 and Fig.3 we plot the result of the numerical calculations of $G_{2}\left(\theta, d, \theta_{A}\right)$ for the Harrison-Zeldovich and "tilted" spectra of the initial perturbations of the metric for different values of $\theta_{A}$. The results of the computations of $G_{2}\left(\theta, d, \theta_{A}\right)$ in the neighborhood of the extrema $\theta \approx \theta_{R}$ can easily be interpreted using the 


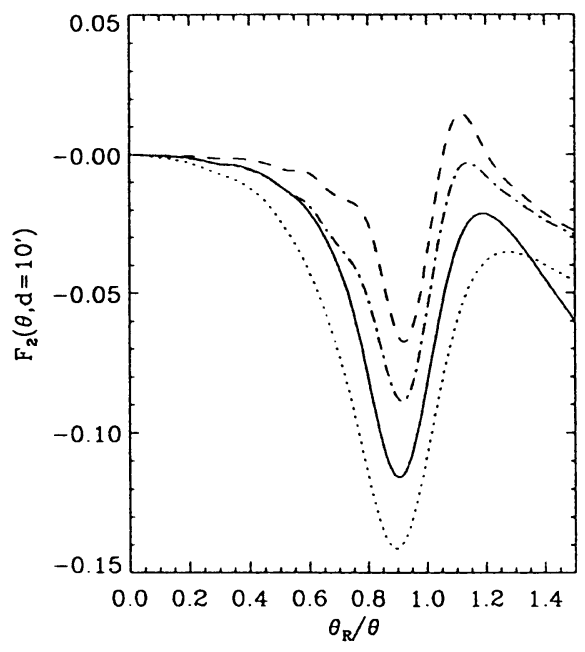

Figure 3: Function $F_{2}\left(\theta, d, \theta_{A}\right)=G_{2}\left(\theta, d, \theta_{A}\right) / G_{2}\left(0, d, \theta_{A}\right)$ for the same model as on Fig.2 $\left(d=10^{\prime}\right)$. We consider the following values of the exponent of the spectrum of primordial perturbations $n$. Solid line for $n=3, \theta_{A}=0.1 \theta_{R}$, Dashed line for $n=3, \theta_{A}=0$, Dotted line for $n=3.5, \theta_{A}=0.1 \theta_{R}$, Dashed-dotted line for $n=3.5, \theta_{A}=0$.

properties of $C(\theta)$ which we have been found in the paper of Jørgensen et al. (1994). We have demonstrated that the LDC effect manifests itself analytically most clearly at the range $\theta \approx \theta_{R}$

$$
G_{2}\left(\theta \approx \theta_{R}, d, \theta_{A}\right) \approx \frac{9 \varepsilon d^{2}}{a(1+\varepsilon)^{1 / 4}} \frac{1}{\theta_{R} \theta_{c}} C_{0}\left(0, \theta_{e f}\right) x\left(1-\frac{5}{18} \beta x^{2}\right),
$$

where $x=\frac{\theta-\theta_{R}}{\theta_{c}}, \epsilon=\left.(1+\nu) \frac{3 \rho_{b}}{4 \rho_{r}}\right|_{z_{r e c}} ; \nu=\frac{2 r_{e q}}{(\sqrt{2}-1) r_{r e c}} ; R$ is an acoustic horizon at $z=z_{\text {rec }}, r_{e q}$ is the Lagrangian radial coordinate of the particle horizon at the redshift of equality of radiation and dark matter densities (note that $\Omega \gg \Omega_{b}$ ), $r_{r e c}$ is the same at the moment of recombination.

One can see that $G_{2}\left(\theta \approx \theta_{R}, d, \theta_{A}\right)$ equal to zero at $\theta=\theta_{R}$ and looks like an almost linear function of $x$ up to the extrema. Further the factor $1-\frac{5}{18} \beta x^{2}$ plays a significant role (see Figures 2 and 3 ). The analogous phenomenon takes place at $\theta \approx 2 \theta_{R}$, however in this range the amplitude of $G_{2}\left(\theta \approx 2 \theta_{R}, d, \theta_{A}\right)$ is very small.

Let us now consider the dependence of the function $G_{2}\left(\theta, d, \theta_{A}\right)$ on the value of the half-width of the antenna-beam $\theta_{A}$. For this purpose we calculated $G_{2}\left(\theta, d, \theta_{A}\right)$ for $\left(\theta_{A} / \theta_{R}\right)^{2}=0.1 ; 0.01$ (see Fig.2). As it is seen on the Fig.2, the peaks are smoothed out for a significant $\theta_{A}$. Thus for the filtering of the effect under consideration we must have both $\theta_{A}, d<\theta_{c}$. 
On Fig. 3 we plot the function $F_{2}\left(\theta, d, \theta_{A}\right)=G_{2}\left(\theta, d, \theta_{A}\right) / G_{2}\left(0, d, \theta_{A}\right)$ for the "tilted" models of the initial power spectrum (see, for example Muciaccia et al., 1993). One can see that the level of modulation depends on the exponent of the spectrum and $\theta_{e f}, \theta_{e f}^{2}=\theta_{A}^{2}+\theta_{c}^{2}$. Besides that, if we have a fixed value of the antenna beam $\theta_{A}$, the depth of the negative peak at $\theta \approx \theta_{R}$ increases when we go from a Harrison-Zeldovich spectrum to the "tilted" one with $n=3.5$. For fixed $n$ we have an analogous feature for increasing $\theta_{A}$. At the same time the strength of the peak of $G_{2}\left(\theta, d, \theta_{A}\right)$ at $\theta \approx \theta_{R}$ decreases (see Fig.2).

Concluding this section we note, that the 4-beam experiment possesses additional possibilities for an investigation of the field of the CMB. As it follows from Fig. 1 in the 4 -beam scheme the directions $\boldsymbol{l}_{1}, \boldsymbol{l}_{2}$ and $\boldsymbol{l}_{3}, \boldsymbol{l}_{4}$ form two bi-ray schemes. Using this fact we introduce the following function

$$
\begin{aligned}
& G_{1}\left(\theta, d, \theta_{A}\right)=\left\langle\left[\frac{T\left(l_{1}\right)-T\left(l_{2}\right)}{T}\right]^{2}\right\rangle-\left\langle\left[\frac{T\left(l_{3}\right)-T\left(l_{4}\right)}{T}\right]^{2}\right\rangle= \\
& =2\left[C\left(\theta-d, \theta_{A}\right)-C\left(\theta+d, \theta_{A}\right)\right] .
\end{aligned}
$$

For the angles $\theta \approx \theta_{R}$ and $\theta \approx 2 \theta_{R}$ at $d \ll \theta_{R}$ the function $G_{1}\left(\theta, d, \theta_{A}\right)$ is strictly connected with the value of the first derivative of the correlation function $C\left(\theta, \theta_{A}\right)$ with respect to the angle $\theta$ :

$$
G_{1}\left(\theta, d, \theta_{A}\right) \approx-4 C^{\prime}\left(\theta, \theta_{A}\right) d,
$$

where $C^{\prime}\left(\theta, \theta_{A}\right) \equiv \frac{d C}{d \theta}$.

One can evaluate the character of the function $G_{1}\left(\theta, d, \theta_{A}\right)$ in the neighborhood of the point $\theta \approx \theta_{R}$. In the paper Jørgensen et al. (1994) we have demonstrated that

$$
G_{1}\left(\theta \approx \theta_{R}, d, \theta_{A}\right) \approx \frac{12 \varepsilon}{a(1+\varepsilon)^{1 / 4}} \frac{d}{\theta_{R}} C_{0}\left(0, \theta_{A}\right)\left(1-\frac{3}{2} x^{2}+\frac{5}{24} \beta x^{4}\right),
$$

where $a=9 \epsilon^{2}+\frac{2+\epsilon}{2(1+\epsilon)^{3 / 2}}$ and $\beta=\frac{3(5-n)}{40}\left(\frac{\theta_{c}}{\theta_{e f}}\right)^{2}$. As one can see from (11), the function $G_{1}\left(\theta=\theta_{R}, d, \theta_{A}\right)$ reaches an extremum at the point $\theta=\theta_{R}$ and has a form of a pronounced peak. This result is confirmed by numerical computations of $G_{1}\left(\theta, d, \theta_{A}\right)$ which are shown on Fig.4. It is seen that in this case the formation of peak-like anomalies takes place at the points $\theta \approx \theta_{R}$ and $\theta \approx 2 \theta_{R}$ where the function $G_{2}\left(\theta, d, \theta_{A}\right)$ has the assymptotics $G_{2}\left(\theta \rightarrow \theta_{R}, d, \theta_{A}\right) \propto\left(\theta-\theta_{R}\right)$ and $G_{2}\left(\theta \rightarrow 2 \theta_{R}, d, \theta_{A}\right) \propto\left(\theta-2 \theta_{R}\right)$.

We have to mention another important property of the functions $G_{1}\left(\theta, d, \theta_{A}\right)$ and $G_{2}\left(\theta, d, \theta_{A}\right)$. As we has shown above, the half-width of the peaks of the function $G_{2}\left(\theta, d, \theta_{A}\right)$ at $\theta \approx \theta_{R}$ is determined by the coefficient $\beta$ (see (8)). For the function $G_{1}\left(\theta, d, \theta_{A}\right)$ the half-width of the peaks is strictly determined by the angle $\theta_{c}$ (see (11)). Thus, using the properties of the functions $G_{1}\left(\theta, d, \theta_{A}\right)$ and $G_{2}\left(\theta, d, \theta_{A}\right)$ at $\theta \approx \theta_{R}$ we can, in principle, get $\theta_{c}$ and $\beta$ after estimation of 


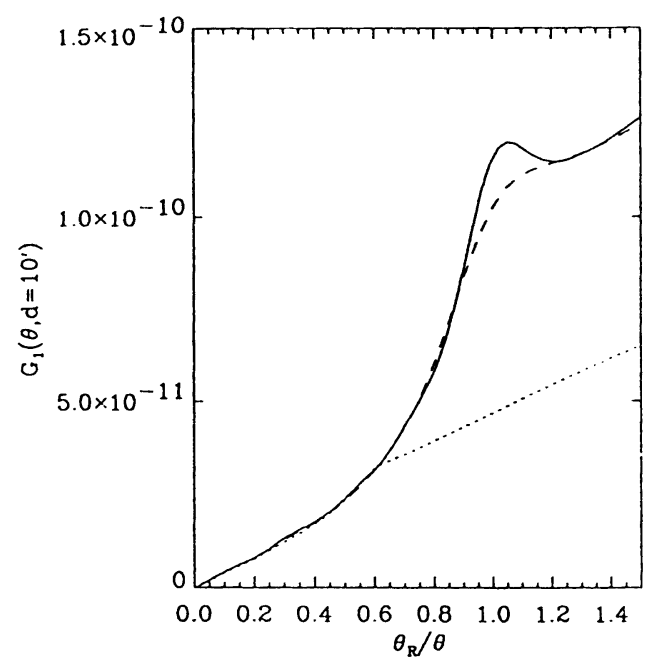

Figure 4: Function $G_{1}\left(\theta, d=10^{\prime}, \theta_{A}\right)$ for the model $\Omega=1 ; \Omega_{b}=0.03 ; h=0.5$; $d=10^{\prime}$ and the Harrison-Zel'dovich spectrum. Solid line for $\theta_{A}=0$, dashed line for $\theta_{A}=0.1 \theta_{R}$, pointed line for $\theta_{A}=0.3 \theta_{R}$

the half-width of the peaks. Both these parameters play an important role in the determination of the statistical properties of the random temperature fluctuations of CMB on the celestial sphere (Zabotin and Naselsky (1985); Sazhin (1985); Bond and Efstathiou (1987)).

\section{Conclusion}

We gave a brief review of a problem of dark matter in the Universe and described a few new techniques of the investigation of the dark matter.

We have considered the peculiarities of the angular correlation function for $\frac{\Delta T}{T}$ at the most interesting angular scales $\theta \simeq 20^{\prime}-2^{\circ}$. We emphasized that the Sakharov oscillations lead (in a CDM model) to appearance of a specific anomalies ("resonances") at the angles $\theta_{R}$ and $\theta_{2 R} \approx 2 \theta_{R}$.

The dependence of the "resonances" on the parameters of the cosmological model and the width of an antenna was investigated. We pointed out the special importance of the first "resonance" at $\theta_{R}$ for the comparison with observational data. This first "resonance" is specific for the model with non-baryonic dark matter and it is absent in the model with baryonic dark matter.

We propose a specific method of filtering of the "resonances" in the correlation function, which is reduced to the direct measure of the second derivative of the correlation function in the vicinity of the "resonance". 


\section{Acknowledgements}

This investigation was supported in part by the Danish Natural Science Research Council through grant 11-9640-1 and in part by Danmarks Grundforskningsfond through its support for an establishment of the Theoretical Astrophysics Center.

\section{References.}

Alcock, C. et al. 1993, Nature 365, 621.

Aubourg, E. et al. 1993, Nature 365, 623.

Bahcall, N.A., 1994, Princeton Observatory Preprint, POP-570.

Bond, Y.R. \& G. Efstathiou: 1987. MNRAS, 226, 655.

Carswell, R.F., Rauch, M., Weymann, R.J., Cooke, A.J., and Webb, J.K., 1994, MNRAS, 268, L1.

Dekel, A. 1993, in the book: 2nd Course, current topics in astrofundamental physics, ed. by . N. Sanchez and A. Zichichi, World Scientific, p. 297.

Dekel, A. 1994, Annual Reviews of Astronomy and Astrophysics 32.

Griest, K. 1994, Particle- and Astro-Physics of Dark Matter, APS Summer Study 94, Snowmass.

Holtzman, J.A., 1989, Ap.J. Suppl., 71

Jørgensen H.E., E.Kotok, P.Naselsky, I.Novikov, 1994, Astronomy and Astrophysics, in press.

Kormendy, J. and G.R.Knapp ; 1987, in the book: Dark Matter in the Universe, Proceedings of the 117-th IAU symposium, ed. by J.Kormendy and G.Knapp, p.XV, D.Reidel Publishing Company

Kolb, E.W., Turner, M.S. 1990, The Early Universe, Addison-Wesley Publishing Company.

Muciaccia, P.F., S. Mei, G. de Gaspers, and N. Vittorio: 1993, Ap.J. 410, L61.

Naselsky, P.D., and I.D. Novikov: 1993a, Ap.J.413,14.

Naselsky, P.D. and I.D. Novikov: 1993b, Proc. of Int.Conf (Erice).

Olive, K.A., Schramm, D.N., Steigman, G., walker, T.P. 1990, Phys. Lett. B, 236, 254.

Paczynski, B. 1986, ApJ., 304, 1.

Pagel, B.E.J. 1990, in the book: Baryonic Dark Matter, eds. Lynden-Bell, D., Gilmore, G., Kluwer, Dardrecht, p. 237.

Pagel, B. 1994a, Light Elements: From Big Bang to Stars, Invited review at Third Intl. Symp. Nuclei in the Cosmos, Gran Sasso, 8-13 July, 1994, M. Busso, R. Gallino \& C. Raiteri (eds.), Amer. Inst. Phys. Publ.

Pagel, B. 1994b, helium in Hii Gegions and Stars, Invited review at ESO/EIPC Workshop on Light Elements, Elba, 23-28 May 1994.

Pagel, B. 1994c, Fine Structure in The main Sequence: Primordial Helium and $\Delta Y / \Delta X$ paper contributed to IAU Symposium 166: Astronomical and Astrophysical Objectives of Sub-milliarcsecond Astronometry, The Hague 
Peebles. P.J.E., I.T. Yu: 1970, Ap.J. 162, 815.

Persic, M., Salucci, P. 1992, MNRAS, 258, p. 14.

Sazhin, M.V.: 1985, MNRAS, 216, 25.

Sakharov, A.D.: 1965, JETP, 49, 345.

Silk, J.: 1968 Ap.J. 151, 459.

Smoot, G.F., 1994, in the book: Present and Future of the Cosmic Microwave Background, ed. by J.L.Sanz, E.Martinez-Gonsalez, L.Cayon. p.67, SpringerVerlag.

Songaila, A., Cowie, L.L., Hogan, C.J., and Rugers, M. 1994, Nature, 368, 599.

Starobinskii, A.A.: Sov.Astronomy Lett. 14 (3), 166, 1988.

Steigman, G., 1994, An Introduction to Cosmological Dark Matter, Preprint, The Ohio State University, Theoretical Astrophysics.

Steigman, G., Tosi, M. 1992, ApJ, 401, 150.

Tyson, J.A. 1994, in the book: Cosmology and Large Scale Structure, Les Houches, August 1993, ed. R. Schaeffer, Elsevier Science Publishers B.V.

Tyson, J.A., Valders, F., and Wenk, R.A. 1990, ApJ, 349, L1.

Watson, R.A., Gutierrez de la Cruz, C.M.: Ap.J. 413, L5, 1993.

Zabotin, N. and P. Naselsky: 1985: Sov. Astron 29, 614.

Zel'dovich, Ya.B. and I.D. Novikov: 1983, The Structure and Evolution of the Universe. (The University of Chicago Press.1983) v.2.

Zwicky, F. 1933, helv. Phys. Acta., 6, 110. 